Itinéraires Itinéraires

Littérature, textes, cultures

2019-1 | 2019

La « renaissance littéraire » africaine en débat

\title{
La « renaissance littéraire africaine » en débat
}

Introduction

Interrogating the African Literary "Renaissance." Introduction

Wilfried Idiatha, Aurélie Journo et Magali Nachtergael

\section{CpenEdition}

Journals

Édition électronique

URL : http://journals.openedition.org/itineraires/6236

DOI : $10.4000 /$ itineraires.6236

ISSN : 2427-920X

Éditeur

Pléiade

Référence électronique

Wilfried Idiatha, Aurélie Journo et Magali Nachtergael, « La « renaissance littéraire africaine » en débat », Itinéraires [En ligne], 2019-1 | 2019, mis en ligne le 24 juillet 2019, consulté le 10 décembre 2020. URL : http://journals.openedition.org/itineraires/6236 ; DOI : https://doi.org/10.4000/itineraires. 6236

Ce document a été généré automatiquement le 10 décembre 2020.

Itinéraires est mis à disposition selon les termes de la licence Creative Commons Attribution - Pas d'Utilisation Commerciale - Pas de Modification 4.0 International. 


\title{
La « renaissance littéraire africaine » en débat
}

\author{
Introduction \\ Interrogating the African Literary "Renaissance." Introduction
}

Wilfried Idiatha, Aurélie Journo et Magali Nachtergael

1 Pour la littérature africaine, le tournant du xxI siècle semble avoir été marqué par une vitalité littéraire et créatrice retrouvée. De nombreux auteurs et autrices africaine-s ont connu une visibilité accrue sur la scène littéraire internationale tandis que sur le continent ont émergé des revues littéraires (Kwani? au Kenya, Saraba au Nigéria, Chimurenga en Afrique du Sud, entre autres), des maisons d'édition locales (Cassava Republic et Kachifo au Nigéria, les récentes éditions fusionnées Ifrikiya au Cameroun ou Hemar au Congo-Brazzaville), ou des collectifs d'écrivains (Femrite en Ouganda) ${ }^{1}$. Parallèlement, des figures de la littérature africaine francophone et anglophone, comme Alain Mabanckou, Chimamanda Ngozi Adichie, Teju Cole, Tayie Selasi, Fatou Diome, In Koli Jean Bofane, ou Léonora Miano, font désormais partie du paysage éditorial globalisé, entraînant dans leur sillage de nouvelles formes d'expressivité littéraires et artistiques (danse, théâtre, performance, musique). Le choix de certains, comme Sami Tchak, de se faire éditer en Afrique témoigne de cette effervescence autour du livre et de la production littéraire sur le continent africain.

2 Ces phénomènes se sont vus relayés dans les médias et par les critiques à travers les expressions de «renaissance» ou de renouveau («renewal») littéraires (Walsh 2003, Schappell et Spillman 2007). D'autres critiques, écrivains ou universitaires, comme Helon Habila, Pius Adesanmi et Chris Dunton, ou encore Paul Zeleza, ont qualifié cette génération de la fin des années 1990 et du début des années 2000 de "troisième génération» d'écrivains africains, tandis que dans le domaine francophone, Abdourahman Waberi (1998) désignait sous le nom d'« enfants de la postcolonie » les écrivains appartenant à la quatrième génération. Ces critiques ont cherché à mettre en lumière les traits qui la différencieraient des générations qui l'ont précédée, sans pour autant passer sous silence les éléments de continuité qui la rattachent à ces dernières. Tous semblent insister sur le caractère cosmopolite ("post-national», pour citer 
Habila) de cette génération, qui explique un rapport à l'identité et aux racines culturelles africaines plus fluide, privilégiant l'idée de multiplicité d'appartenances à celle d'hybridité. En outre, si le thème de la migration occupe une place toujours importante, de nouveaux thèmes ont fait leur apparition, tels quela question de genre ou LGBTQIA ou les phénomènes de radicalisation et de terrorisme global, entre autres. Enfin, des genres souvent sous-représentés et considérés comme mineurs se sont vus investis ou ré-investis par de nombreux écrivains, que l'on songe à la science-fiction, au roman sentimental ou au roman policier.

Si de nombreux chercheurs et critiques ont pu déceler dans la fin $\mathrm{du} \mathrm{xx}^{\mathrm{e}}$ siècle et le début du XXI ${ }^{\mathrm{e}}$ siècle un tournant dans la production littéraire africaine, c'est aussi parce que cette période a été marquée par un certain nombre de changements d'ordre politique, social ou technologique qui ont modifié les conditions de production littéraire. On peut citer tout d'abord les mouvements de "décompression autoritaire " (Bayart 1991) qui ont mené au retour au multipartisme au Kenya ou à la fin de la dictature militaire au Nigéria, la disparition du régime de Mobutu Sese Seko au Zaïre aujourd'hui République démocratique du Congo, mais aussi la fin de la guerre civile au Sierra Leone ou de l'apartheid en Afrique du Sud, évolutions politiques et sociales : ces bouleversements socio et géopolitiques ont contribué à un climat plus propice à la création littéraire, mais aussi à la modification de la réception des œuvres. On peut à cet égard évoquer la place qu'occupe la critique littéraire africaine qui semble prendre un nouvel élan aujourd'hui et, bien évidemment, la place de ces littératures dans le monde académique, à l'image de l'université Omar Bongo de Libreville qui s'est dotée d'un département de littératures africaines au sein duquel la « littérature gabonaise » est désormais une filière à part entière.

4 Le développement d'Internet a également joué un rôle important en ce qu'il est rapidement apparu comme une plateforme de diffusion et de démocratisation de la pratique littéraire, mais aussi comme un lieu d'échange et de sociabilité où ont pu se développer des communautés littéraires qui ont en partage moins l'appartenance à un espace commun que des aspirations et des préoccupations partagées. Enfin, il faut souligner le rôle important qu'ont joué les prix littéraires comme le prix Caine, créé en 2000 ou l'historique Grand Prix littéraire d'Afrique noire (le « Goncourt africain »), mais aussi les nombreux festivals littéraires consacrés régulièrement à l'Afrique, dans la visibilité accrue des écrivains du continent.

5 L'objectif de ce numéro est donc de s'interroger sur ce que l'on entend lorsque l'on parle de "renaissance littéraire» à propos de la production littéraire africaine contemporaine, afin de mesurer et de penser conjointement les phénomènes de rupture et de permanence qui la sous-tendent.

6 Les articles de ce numéro s'intéressent tout à la fois aux changements et aux permanences thématiques, au renouveau générique et au réinvestissement de certains genres. Ils proposent des lectures qui témoignent de la variété des pratiques littéraires contemporaines et de la spécificité des conditions socio-politiques dans lesquelles elles se situent, et se penchent sur l'évolution de «l'espace des possibles» (Bourdieu) en s'intéressant à l'émergence d'institutions littéraires continentales (prix, maisons d'édition, revues, collectifs d'écrivains). En effet, l'influence et le pouvoir prédominants des « centres littéraires» décrits par Pascale Casanova dans La République mondiale des lettres persistent et se confondent désormais avec la notion de globalisation, décrite par un collectif de penseurs auquel Achille Mbembe a pris part, de sorte que global est à 
entendre à un niveau continental, mais aussi au-delà à travers les interactions diasporiques. Ces nouvelles structures mettent au jour le développement de réseaux de sociabilité littéraire panafricains et nous invitent à repenser et à complexifier le paradigme qui oppose centres et marges de l'espace littéraire mondial et conçoit leur rapport selon des forces désormais multilatérales.

\section{Questions de genre(s)}

7 Les articles d'Hervé Tchumkam et de Pierre Leroux ouvrent ce numéro et reviennent sur la façon dont certains auteurs investissent et renouvellent les genres policier (Jean Roger Essomba et Achille Ngoye) et sentimental (Tendai Huchu).

S'appuyant sur les analyses de Giorgio Agamben, de Judith Butler et de Guy Debord, Hervé Tchumkam propose une lecture des romans policiers du Congolais Achille Ngoye et du Camerounais Jean Roger Essomba qui se situe à l'intersection de l'esthétique et du politique. Il met en évidence la façon dont ces fictions mêlent polar de l'immigration, paranormal et Bildungsroman politique. Chez Achille Ngoye le genre policier revêt une fonction sociale et pédagogique autour de la question du retour d'exil et de l'autopsie possible des États postcoloniaux tandis que le roman de Jean-René Essomba, en ce qu'il "met à nu les logiques capitalistes et impérialistes qui installent durablement les citoyens africains dans l'insécurité et la terreur» et invite à une réflexion sur «l'humanitaire et sur le totalitarisme local (africain) et mondial», remplit une fonction philosophique.

Pierre Leroux, dans son article sur le premier roman du Zimbabwéen Tendai Huchu, The Hairdresser of Harare, revient sur un autre genre populaire, le roman sentimental, investi par la thématique de l'homosexualité, relativement peu traitée dans la littérature zimbabwéenne. Il montre comment «la représentation de l'homosexualité et des questions de genre (gender) est indissociable [...] du travail sur les genres littéraires populaires ", et permet, dans un récit qui s'appuie sur des ressorts génériques précis et délègue le débat sur l'homosexualité à plusieurs voix narratives, de mettre au jour divers aspects d'une société zimbabwéenne en crise.

10 La question de l'identité genrée est également au cœur de l'article d'Ashwiny O Kistnareddy. Dans sa lecture des romans de Fatou Diome et de Léonora Miano, elle envisage la façon dont celles-ci proposent de reconceptualiser et de reconstruire les masculinités noires dans un contexte postcolonial (la France) et diasporique, en articulant et en arrimant la question de la / des masculinité(s) noire(s) à celles relatives à l'exil, au retour et à la nostalgie.

\section{Renaissance linguistique et pratiques littéraires}

11 Pierre Boizette propose une lecture historique et linguistique de la notion de renaissance africaine en la resituant dans les débats autour de la question de la langue d'écriture et de la revalorisation des langues africaines des années 1960, et le rôle de la promotion des langues africaines dans cette renaissance. Cette dimension plurilingue est au cœur de l'engagement du romancier kényan Ngugi wa Thiong'o mais aussi de la pensée du philosophe ghanéen Kwasi Wiredu: le lien à la langue hégémonique, l'anglais, loin de cristalliser un rejet pur et simple, permet d'approcher le "rêve 
panafricain " et de constituer une communauté dans laquelle une conversation peut prendre place. Cet aspect transnational favorise les dialogues intercontinentaux mais aussi les sociabilités littéraires qui participent à la production, la circulation et la légitimation des œuvres en dehors du pays d'origine des auteur.trices.

Dans le domaine francophone, la théorisation de la pratique littéraire peut aussi, comme chez Léonora Miano et Alain Mabanckou, se constituer à partir d'une définition identitaire, qu'elle soit postcoloniale, diasporique ou liée à l'expérience de l'identité noire. Dans cette confraternité linguistique qu'est la langue française, Josefina Bueno Alonso analyse L'Afrique qui vient à l'aune de l'afrodescendance, dans le sillage de la "génération transcontinentale " décrite par Abdourahman Waberi. Ainsi, Miano et Mabanckou adoptent une position politique qui appelle au dépassement de la «francophonie» et croise les enjeux de la diaspora, des migrations et de l'identité noire. Cette production littéraire non pas tant activiste qu'afropolitique est celle également prônée par le Camerounais Achille Mbembe ou encore portée par le concept d'« afrotopie » développé par Felwine Sarr en 2016.

\section{Mémoire collective et sociabilités littéraires : le cas de la revue Kwani? (Kenya)}

13 Le rôle des revues dans la constitution des communautés de lecteurs et d'auteurs mais aussi dans la promotion de certaines pratiques littéraires est bien souvent déterminant. Dans son étude de Kwani?, revue et plateforme éditoriale kényane, Kate Wallis s'intéresse à l'espace d'expression d'une « mémoire populaire collective » que celle-ci a ouvert au Kenya. Elle analyse l'attention particulière portée à l'historiographie et à la mémoire historique par les membres du Kwani Trust, dont font partie Andia Kisia, Parselelo Kantai, Billy Kahora et Yvonne Adhiambo Owuor, ainsi que la façon dont leurs textes œuvrent à la matérialisation d'une forme de mémoire populaire. Mais comment combiner histoire populaire et nationale, en somme quelle histoire écrire, pour quelle mémoire ? Le texte de la conférence que le rédacteur en chef de la revue Billy Kahora a donnée à Paris en 2017 permet d'éclairer, de l'intérieur, les enjeux et le contexte, tant politiques que littéraires, qui a vu l'apparition de la revue au début des années 2000. Il y présente les diverses facettes de la revue mais aussi les réseaux de sociabilité littéraires qui la sous-tendent, et montre le rôle que celle-ci, par ses publications et les événements qu'elle organise, a joué dans « la renaissance littéraire » continentale.

Les varias associés à ce dossier prolongent les contours de la «renaissance littéraire africaine " notamment en explorant la représentation de la contemporanéité dans l'écriture romanesque d'Afrique. L'article de Pierre-Yves Dufeu s'attache à interroger le rôle du vêtement dans La Saison de l'ombre de Léonora Miano, et le regard mulongo qui se déploie dans ce récit situé à l'époque de la traite négrière. Lorsque l'écrivain congolais Pius Ngandu Nkashama publie Un jour de grand soleil sur les montagnes de l'Éthiopie, il s'inspire d'une révolte lycéenne contre le régime éthiopien dans les années 1970, tout en mêlant sa propre expérience du Congo-Zaïre sous Mobutu. Dans son article portant sur la folie et le trauma, Aurélia Mouzet montre en quoi les psychoses personnelles décrites dans ce récit sont une métaphore de la folie dictatoriale qui marque les individus autant que la collectivité. Enfin, l'article d' Emmanuel Ndour se déplace du côté des Amériques, où l'auteur haïtien Dany Laferrière dialogue avec un jeune immigré camerounais, rencontré par hasard dans une rue de 
Montréal, et prodigue, dans un enchevêtrement de souvenirs, des conseils au nouvel arrivant. Ndour pose l'hypothèse d'une véritable éthique du déchiffrement à l'œuvre, dans un échange constant avec sa propre altérité et dans des "subjectivités alternatives ».

\section{BIBLIOGRAPHIE}

Adesanmi, Pius et Dunton, Chris (dir.), 2008, « Nigeria's Third-Generation Novel: Preliminary Theoretical Engagements », Research in African Literatures, vol. $39 \mathrm{n}^{\circ} 2$.

Bayart, Jean-Francois, 1991, « La Baule, et puis après ? ", Politique africaine, nº 43, p. 5-20.

Bourdieu, Pierre, [1992] 1998, Les Règles de l'art. Genèse et structure du champ littéraire, Paris, Seuil.

Casanova, Pascale, [1999] 2008, La République mondiale des lettres, Paris, Seuil.

Habila, Helon, 2012, « Introduction », dans H. Habila (dir.), The Granta Book of the African Short Story, Londres, Granta Books, p. vii-xv.

Mbembe, Achille et Sarr, Felwin (dir.), 2017, Écrire l'Afrique-Monde. Les Ateliers de la pensée, Paris, Philippe Rey.

Sarr, Felwin, 2016, Afrotopia, Paris, Philippe Rey.

Schappell, Elissa et Spillman Rob, 2007, « The Continental Shelf », Vanity Fair, juillet 2007, p. 118-197.

Waberi, Abdourahman, 1998, «Les enfants de la postcolonie : esquisse d'une nouvelle génération d'écrivains francophones d'Afrique noire ", Notre librairie, $\mathrm{n}^{\circ}$ 135, p. 8-15.

Walsh, Declan, 2003, « Kenya's Literary Renaissance Gives a Voice to Urban Living in Nairobi », The Independent, 11 octobre 2003.

Zeleza, Paul, 2007, « Colonial Fictions: Memory and History in Yvonne Vera's Imagination », Research in African Literatures, vol. 38, $\mathrm{n}^{\circ}$ 2, p. 9-21.

\section{NOTES}

1. Ce numéro d'Itinéraires prolonge les réflexions qui se sont tenues lors d'une journée d'étude intitulée $L a$ "renaissance littéraire " africaine anglophone en débat qui a eu lieu à l'université Paris 13 le 5 juillet 2017. 


\section{AUTEURS}

WILFRIED IDIATHA

Université Paris 13

AURÉLIE JOURNO

Université Paris 13, Pléiade (EA 7338)

MAGALI NACHTERGAEL

Université Paris 13, Pléiade (EA 7338) 